CONGENITAL HEART DISEASE

\title{
Permanent junctional reciprocating tachycardia in children: a multicentre study on clinical profile and outcome
}

\author{
G Vaksmann, C D'Hoinne, V Lucet, S Guillaumont, J-M Lupoglazoff, A Chantepie, \\ I Denjoy, E Villain, F Marçon
}

See end of article for authors' affiliations

Correspondence to: Dr Guy Vaksmann, Polyclinique de la Louviere, 69 rue de la Louviere, 59800, Lille, France; guy. vaksmann@wanadoo.tr

Accepted 7 April 2005 Published Online First 7 April 2005

\begin{abstract}
Objectives: To investigate the clinical profile, natural history, and optimal management of persistent or permanent junctional reciprocating tachycardia (PJRT) in children.

Methods and results: 85 patients meeting the ECG criteria for PJRT were enrolled in a retrospective multicentre study. Age at diagnosis varied from birth to 20 years (median 3 months). Follow up ranged from 0.1 to 26.0 (median 8.2) years. At the time of referral, 24 of 85 patients (28\%) had congestive heart failure that was resolved with medical treatment in all patients. Eighty three patients received drug treatment initially. Amiodarone and verapamil were the most effective with a success rate of $84-94 \%$ alone or in association with digoxin. Radiofrequency ablation of the accessory pathway was performed in 18 patients. There was a trend for a relation between age at ablation and the result of the procedure, failures being more common in younger patients (three of six procedures in younger and 15 of 18 in older children were successful; $p=0.14$ ). Two patients with persistent left ventricular dysfunction on echocardiography but with no symptoms of congestive heart failure died suddenly one month and three years after diagnosis. PJRT resolved spontaneously in 19 patients (22\%). Age at diagnosis of PJRT was not a predictor of spontaneous resolution.

Conclusions: PJRT is a potentially lethal arrhythmia in children with tachycardia induced cardiomyopathy. Spontaneous resolution of tachycardia is not uncommon. Antiarrhythmic treatment is often effective. Radiofrequency ablation should be performed in older children or when rate is not controlled, especially in patients with persistent left ventricular dysfunction.
\end{abstract}

$\mathrm{P}$ ersistent or permanent junctional reciprocating tachycardia (PJRT) is an uncommon arrhythmia first described by Coumel et al, ${ }^{1}$ characterised by an incessant orthodromic tachycardia with anterograde conduction over the atrioventricular node and by retrograde conduction via an accessory pathway usually located in the posteroseptal region with slow and decremental conduction..$^{2-4}$. The arrhythmia is commonly incessant from birth or infancy, even though it may not be recognised until adulthood. ${ }^{5}$ Its persistence over a long period of time may lead to a tachycardia induced cardiomyopathy that is reversible with rate control. ${ }^{6-10}$ PJRT is thought not to have the spontaneous resolution characteristics of other atrioventricular re-entry tachycardias in infancy but spontaneous resolution has been reported in some patients. ${ }^{8}$ The arrhythmia is considered to be usually refractory to drug treatment and various non-pharmacological approaches have been used to prevent tachycardia or for rate control. Over the past few years radiofrequency catheter ablation of the accessory pathway has been reported to be very effective and safe as definitive treatment of PJRT. ${ }^{11-14}$ Although most patients having had catheter ablation had reach adulthood, it has been proposed that the technique should be considered early in the management of these patients. ${ }^{14-16}$ However, because of the rareness of the reports on the natural history of PJRT, little is known about the clinical course of this chronic tachycardia. Single institutional experience of PJRT usually is small, and generally accepted guidelines on how to manage these patients are still lacking. On the basis of a multi-institutional experience reporting the largest population of patients with PJRT, we attempted to determine the clinical profile, natural history, and optimal management of this uncommon arrhythmia.

\section{METHODS}

\section{Patient selection}

This multicentre retrospective study was conducted at seven French paediatric institutions caring for paediatric arrhythmias. The participating centres and the number of contributed patients are as follows: Les loges en Josas (27 patients), Lille (18 patients), Montpellier (13 patients), Nancy (12 patients), Paris (Necker Hospital, 5 patients; Robert Debré Hospital, 6 patients), and Tours (4 patients). Twenty one of the 85 patients have been the subjects of previous reports. ${ }^{9}{ }^{10}$ A questionnaire addressing the patients' clinical course, treatment, and follow up was given to each centre to complete. Patients were enrolled if they presented with PJRT before their 20th birthday. Follow up of each patient had to be longer than one year unless the affected patient died earlier.

Diagnosis of PJRT was based on surface ECG criteria including the presence of an almost incessant narrow QRS tachycardia with negative $\mathrm{P}$ waves in inferior leads, a $\mathrm{P}^{\prime} \mathrm{R}$ interval shorter than the $\mathrm{RP}^{\prime}$ interval, and an atrioventricular ratio of 1:1. During episodes of sinus rhythm the PR interval had to be normal with no evidence of a delta wave. During tachycardia, no episode of functional atrioventricular block could be documented on 24 hour ambulatory recordings or vagal manoeuvres.

All patients were followed up as outpatients. An intracardiac electrophysiological study was performed in 21 patients with subsequent catheter ablation attempted in 18 .

\section{Criteria for treatment effectiveness}

The results of treatment were assessed by repeated ECGs and 24 hour ambulatory recordings. Treatment success was 
defined as conversion to stable permanent sinus rhythm or to predominant sinus rhythm alternating with non-sustained $(<30$ seconds) low rate PJRT $(<110$ beats/min $)$ with normal left ventricular function on echocardiography. Partial success was defined as a significant decrease in heart rate (PJRT rate reduction $>30 \%$ ) associated with periods of sustained sinus rhythm on Holter monitoring and normalisation of left ventricular function on echocardiography if dysfunction was initially present. Failure was defined as a non-significant decrease of heart rate or persistence of left ventricular dysfunction if initially present. Spontaneous resolution of PJRT was defined as no recurrence of PJRT after cessation of antiarrhythmic treatment with permanent sinus rhythm on Holter monitoring and an exercise test in patients older than 8 years.

\section{Statistical analysis}

Data were compared by Student's $t$ test for continuous variables. Relative frequencies observed between groups of patients were compared by Fisher's exact test.

\section{RESULTS}

\section{Patient characteristics at presentation}

The 85 patients (43 female and 42 male patients) were admitted for incessant tachycardia in 1966-1998. The initial age at diagnosis varied from birth to 20 years (median age 3 months); 57 of 85 patients presented before 1 year of age and 19 of them had a history of intrauterine tachycardia (three with hydrops fetalis), although the type of tachycardia could not be ascertained from the fetal echocardiogram (fig l). One patient with Williams syndrome had a mitral valve prolapse with grade 2 mitral regurgitation. No other patient had a structural heart defect.

At the time of referral, 24 of 85 patients $(28 \%)$ presented with varying degrees of congestive heart failure. Congestive heart failure was more common in infants (2l of $57(37 \%)$ ) than in children older than 1 year (three of $28(11 \%)$, $\mathrm{p}<0.05)$. Infants had a higher tachycardia rate than did older children (221 (40) v 180 (44) beats/min, p < 0.01). However, the ventricular rate in those patients with congestive heart failure was not significantly different from that in patients without congestive heart failure $(217$ (30) $v$ 205 (50) beats/min, $\mathrm{p}>0.05$ ).

\section{Follow up and treatment}

Follow up ranged from 0.1 to 26.0 (median 8.2) years. Of all patients, two never received drug treatment: one because of low rate tachycardia of 100 beats/min, normal left ventricular function, and absence of symptoms; and the other because of primary radiofrequency ablation procedure and with a well tolerated tachycardia of 150 beats/min.

Eighty three patients received drug treatment initially. Among them, only 17 were managed with one single antiarrhythmic drug, whereas 66 received at least two consecutive antiarrhythmic agents. None received more than one antiarrhythmic drug simultaneously, except in

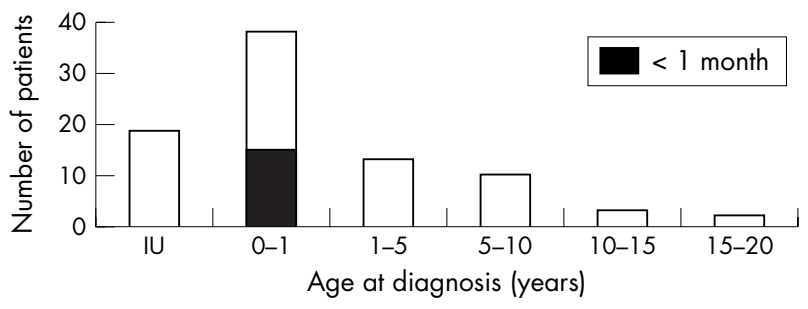

Figure 1 Distribution of patients by age at diagnosis of tachycardia. IU, in utero diagnosis.

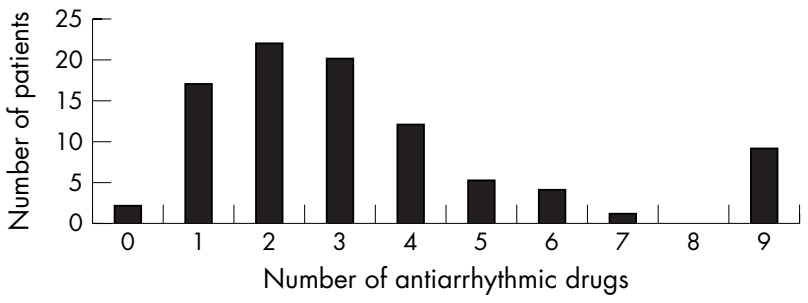

Figure 2 Number of concomitant or consecutive antiarrhythmic agents given to the patients over the whole study period.

combination with digoxin. Of these, 24 even received consecutively four to nine antiarrhythmic drugs (fig 2).

Figure 3 shows a comparison of the effectiveness (complete and partial success) of the antiarrhythmic agents used. The reported initial success rate was highest for amiodarone and verapamil, which were effective or partially effective alone or in association with digoxin in $84-94 \%$ of patients. Verapamil was given if patients were older than 2 years. Digoxin alone had a success rate of $52 \%$. PJRT relapsed after initial success in six patients: four were receiving digoxin, one received digoxin and amiodarone, and one received verapamil. The four patients with relapse while taking digoxin were successfully treated with amiodarone (three patients) or verapamil (one patient). The other two patients underwent successful radiofrequency ablation of their accessory pathway.

Treatment side effect occurred in 12 patients. Two had chemical evidence of hyperthyroidism and four had chemical evidence of hypothyroidism. None had clinical manifestations of thyroid dysfunction and laboratory values normalised after cessation of the drug. Photosensitivity occurred in three other patients who received amiodarone. Corneal microdeposits were found in only one patient receiving amiodarone but were not systematically searched. Two patients receiving flecainide or propafenone had posttachycardic pauses on Holter monitoring of more than three seconds suggesting sinus node dysfunction.

Radiofrequency ablation of the accessory pathway was performed in 18 patients by experienced operators from four institutions in 1988-1998 for a total of 24 procedures. Median age at ablation was 17.5 years (range $4-25$ years). Ablation was successful at the first attempt in 14 patients including the patient with mitral valve prolapse, at the second attempt in three patients, and at the fourth attempt in one patient. There was a trend for a relation between age at ablation and the result of the procedure, failures being more frequent in younger patients. Three of six procedures performed in children younger than 10 years were successful compared with 15 of 18 procedures performed in older children $(\mathrm{p}=0.14)$.

When initially present, congestive heart failure resolved with medical treatment in all patients. However, two patients with a persistent decreased left ventricular ejection fraction on echocardiography, but no symptoms of congestive heart failure died, suddenly. The first one was a 4 year old boy who had pulmonary oedema at admission. He was treated with amiodarone and died one month after the onset of treatment despite rate control and improvement of clinical symptoms and of left ventricular ejection fraction. The second one was a 9 year old girl who received digoxin for three years and was thought to be in good control under treatment but with persistent left ventricular dilatation on echocardiography with a left ventricular ejection fraction of $45 \%$.

PJRT resolved spontaneously in 19 patients (22\%) 5.4 years (two months to 16 years) after diagnosis of tachycardia. Median follow up after spontaneous resolution was 2.2 years 


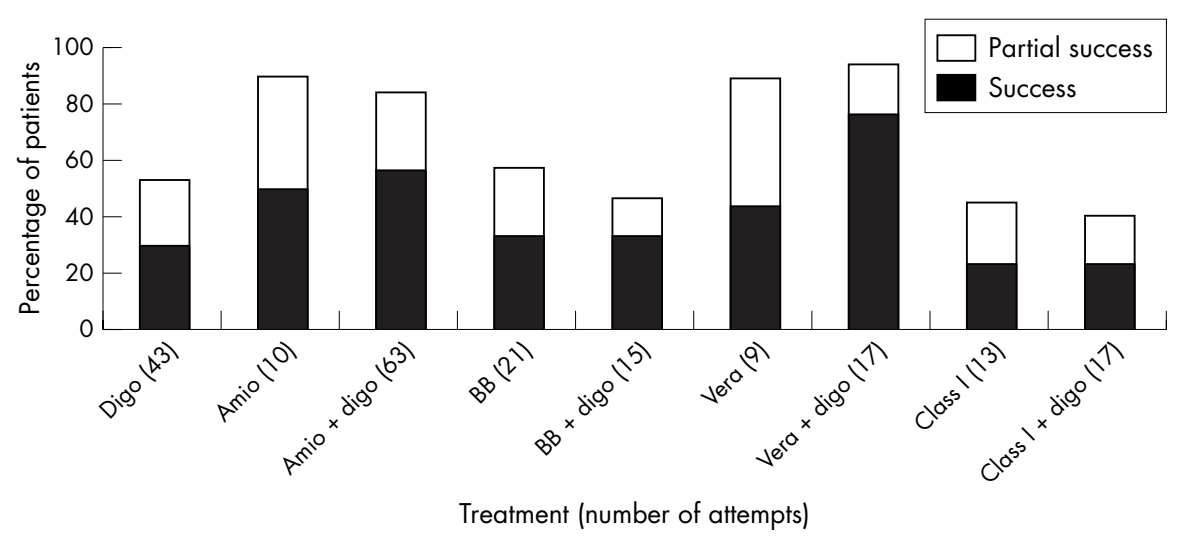

Figure 3 Effectiveness of the antiarrhythmic agents. Amio, amiodarone; BB, $\beta$ blockers; Class I, class I antiarrhythmic agents; Digo, digoxin; Vera, verapamil.

(range 0.3-11.9 years). Age at diagnosis of PJRT was not a predictor of spontaneous resolution. Spontaneous resolution occurred in 14 of $57(25 \%)$ of patients younger than 1 year and in five of $28(18 \%)$ of patients older than 1 year at diagnosis (not significant).

\section{Status at last follow up}

At last follow up, 36 patients (42\%) were free of PJRT on Holter monitoring or exercise testing and free of pharmacological treatment, including the 19 patients who had spontaneous resolution and the 17 with successful radiofrequency ablation. Twenty six patients $(31 \%)$ were also free of PJRT on Holter monitoring but were still treated with antiarrhythmic drugs. Five patients $(6 \%)$ had a low rate PJRT or had an initially incessant tachycardia that became nonsustained and were not treated, among them the patient who was never treated. Only 18 patients $(21 \%)$ had PJRT while receiving drugs, among them the two patients who died suddenly. None of the remaining 16 patients had clinical symptoms or impairment of left ventricular function on echocardiography.

\section{DISCUSSION}

This multicentre study, having collected data on a large number of patients with PJRT, confirmed some findings and brought to light some new characteristics useful for the management of this rare arrhythmia.

\section{Clinical profile}

PJRT may be diagnosed at any age during childhood but is diagnosed in a majority of patients during infancy or prenatally. Symptoms of congestive heart failure were more common among younger patients before the introduction of medical treatment. Patients who are older at diagnosis of PJRT probably have had unrecognised slower tachycardia that was less sustained during early childhood, explaining their better tolerance and their late diagnosis. Congestive heart failure always resolved with antiarrhythmic treatment. Nevertheless, two patients with persistent left ventricular dysfunction on echocardiography, although clinically improved by treatment, died suddenly one month and five years after diagnosis.

Unlike accessory pathways of the Wolff-Parkinson-White syndrome in children that are associated with a structural heart defect in about one third of patients, ${ }^{17-21}$ accessory pathways of PJRT are generally isolated. Only one patient of this series with the Williams syndrome had a structural heart defect. In addition, no patients in two other large published series of 53 patients had a structural cardiac anomaly. ${ }^{822}$

One of the main findings of the present study is the relatively high rate of spontaneous resolution of PJRT, which exceeded $20 \%$. In contrast with observations in smaller series of paediatric patients reported so far, spontaneous resolution of PJRT was not so uncommon. ${ }^{822}$ Such resolution happened sometimes many years after diagnosis of tachycardia.

Another interesting finding of this study is the remarkable effectiveness of some antiarrhythmic drugs against arrhythmia, especially amiodarone and verapamil, which had a success rate of more than $80 \%$. Even patients receiving digoxin alone had a success rate of more than $50 \%$. In our series, radiofrequency ablation had a greater success rate in older patients. In addition, although radiofrequency ablation can be performed at any age, even in infancy, complications in this age group are more common. ${ }^{23-25}$ Furthermore, experimental data have shown that radiofrequency lesions appear to increase in size with somatic growth, with replacement of normal myocytes with fibrous and elastic tissue forming a potential arrhythmogenic substrate. ${ }^{26}$ Thus, considering the high success rate of antiarrhythmic drugs, we recommend that patients with PJRT be treated initially with amiodarone or verapamil in combination with digoxin. The usual effectiveness of these drugs may allow delay of radiofrequency ablation until patients have grown adequately.

\section{Study limitations}

Firstly, the present study was a retrospective and multicentre investigation. However, it enrolled a large number of patients with relatively uniform management. In all the centres, medical treatment was tried first, especially with younger patients, and radiofrequency ablation was a first choice procedure for only one patient. Ablation was undertaken in the other patients after failure of medical treatment or occurrence of side effects or on reaching adulthood.

Secondly, electrophysiological study was not mandatory for patient inclusion. However, ECG and Holter monitoring studies allowed the exclusion of the diagnosis of ectopic atrial tachycardia. Intranodal re-entry, in its rarer form (fast-slow), is another possibility but such a tachycardia is very uncommon in children. We do not believe that the absence of electrophysiological study for patient inclusion changed the conclusions of our study.

Thirdly, an exercise test was not mandatory to consider that antiarrhythmic treatment was successful. However, the goal of treatment was not to obtain permanent sinus rhythm but to avoid sustained high frequency ( $>110$ beats/min) tachycardia on Holter monitoring. However, children older than 8 years who did not receive antiarrhythmic treatment underwent an exercise test to confirm either spontaneous resolution of the tachycardia or success of radiofrequency ablation. 


\section{Conclusion}

PJRT is a potentially lethal arrhythmia in children with tachycardia induced cardiomyopathy. Although rarely reported, spontaneous resolution is not uncommon. Antiarrhythmic treatment is often effective, especially with amiodarone and verapamil. Radiofrequency ablation should be reserved for older patients but may be indicated even in the very young when the rate is not controlled and especially in patients with persistent left ventricular dysfunction.

\section{Authors' affiliations}

G Vaksmann, C D'Hoinne, Department of Paediatric Cardiology,

Cardiological Hospital, Lille, France

V Lucet, Department of Paediatric Dysrhythmias, Le chateau des côtes, Les Loges en Josas, France

S Guillaumont, Department of Paediatric Cardiology, Montpellier,

France

J-M Lupoglazoff, Department of Paediatric Cardiology, Robert Debré Hospital, Paris, France

A Chantepie, Department of Paediatric Cardiology, Trousseau Hospital, Tours, France

I Denjoy, Department of Electrophysiology, Lariboisiere Hospital, Paris, France

E Villain, Department of Paediatric Cardiology, Necker Hospital, Paris, France

F Marçon, Department of Paediatric Cardiology, Paediatric Hospital, Nancy, France

\section{REFERENCES}

1 Coumel P, Cabrol C, Fabiato A, et al. Tachycardie permanente par rythme réciproque. I. Preuves du diagnostic par stimulation auriculaire et ventriculaire. Arch mal Coeur 1967;60:1830-64.

2 Gallagher JJ, Sealy WC. The permanent form of junctional reciprocating tachycardia: further elucidation of the underlying mechanism. Eur J Cardiol 1978;8:413-30.

3 Critelli G, Gallagher JJ, Monda V, et al. Anatomic and electrophysiologic substrate of the permanent form of junctional reciprocating tachycardia. J Am Coll Cardiol 1984;4:601-10.

4 Farre J, Ross E, Weiner I, et al. Reciprocating tachycardias using accessory pathways with long conduction times. Am J Cardiol 1979;44:1099-109.

5 Critelli G, Gallagher JJ, Thiene G, et al. The permanent form of junctional reciprocating tachycardia. In: Benditt DG, Benson DW, eds. Cardiac preexcitation syndromes. Boston: Martinus Nijhoff, 1986:233-53.

6 Packer DL, Bardy GH, Worley SJ, et al. Tachycardia-induced cardiomyopathy: a reversible form of left ventricular dysfunction. Am J Cardiol 1986;57:563-70.

7 Cruz FE, Cheriex EC, Smeets JL, et al. Reversibility of tachycardia-induced cardiomyopathy after cure of incessant supraventricular tachycardia. J Am Coll Cardiol 1990;16:739-44.
8 Lindinger A, Heisel A, Von Bernuth G, et al. Permanent junctional re-entry tachycardia: a multicenter long-term follow-up study in infants, children and young adults. Eur Heart J 1998;19:936-42.

9 Schleich JM, Vaksmann G, Khanoyan P, et al. Les tachycardies jonctionnelles permanentes par rythme réciproque chez l'enfant et l'adolescent: efficacité du traitement médicamenteux. Arch Mal Coeur 1992;85:553-9.

10 Lucet V, Do Ngoc D, Sidi D, et al. Medical treatment and long-term development of permanent reciprocal tachycardia in children: apropos of 10 cases followed for 11 years. Arch Mal Coeur 1985;78:210-6.

11 Smith RT, Gillette PC, Massumi A, et al. Transcatheter ablative techniques for treatment of the permanent form of junctional reciprocating tachycardia in young patients. J Am Coll Cardiol 1986;8:385-90.

12 Haissaguerre $M$, Montserrat $P, W$ arin JF, et al. Catheter ablation of left posteroseptal accessory pathways and of long $\mathrm{RP}^{\prime}$ tachycardias with a right endocardial approach. Eur Heart J, 1991;12:845-59.

13 Critelli G, Gallagher JJ, Perticone F, et al. Transvenous catheter ablation of the accessory atrio-ventricular pathway in the permanent form of junctional reciprocating tachycardia. Am J Cardiol 1985;55:1639-40.

14 Gaita F, Haissaguerre M, Giustetto C, et al. Catheter ablation of permanent junctional reciprocating tachycardia with radiofrequency current. J Am Coll Cardiol 1995;25:648-54.

15 Balaji S, Gillette PC, Case CL. Successful radiofrequency ablation of permanent junctional reciprocating tachycardia in an 18-month-old child. Am Heart J 1994;127:1420-1.

16 Aquinaga L, Primo J, Anguera I, et al. Long-term follow-up in patients with the permanent form of junctional reciprocating tachycardia treated with radiofrequency ablation. Pacing Clin Electrophysiol 1998;21:2073-8.

17 Becquart J, Vaksmann G, Becquart V, et al. Pronostic du syndrome de WolffParkinson-White chez le nourrisson: a propos de 31 observations. Arch Mal Coeur 1988;81:695-700.

18 Giardina ACV, Ehlers KH, Engle MA. Wolff-Parkinson-White syndrome in infants and children. A long-term follow-up study. Br Heart J 1972;34:839-46.

19 Mantakas ME, McCue CM, Miller WW. Natural history of WolffParkinson-White syndrome discovered in infancy. Am J Cardiol 1978:41:1097-103.

20 Deal BJ, Keane JF, Gillette PC, et al. Wolff-Parkinson-White syndrome and supraventricular tachycardia during infancy: management and follow-up. J Am Coll Cardiol 1985;5:130-5.

21 Perry JC, Garson A Jr. Supraventricular tachycardia due to Wolff-ParkinsonWhite syndrome in infants and children: early disappearance and late recurrence. J Am Coll Cardiol 1990;16:1215-20.

22 Dorostkar PC, Silka MJ, Morady F, et al. Clinical course of persistent junctional reciprocating tachycardia. J Am Coll Cardiol 1999;33:366-75.

23 Weindling SN, Saul JP, Walsh EP. Efficacy and risks of medical therapy for supraventricular tachycardia in neonates and infants. Am Heart $J$ 1996;131:66-72

24 Van Hare GF, Witherell CL, Lesh MD. Follow-up of radiofrequency catheter ablation in children: results in 100 consecutive patients. J Am Coll Cardiol 1994;23:1651-9.

25 Kugler D. Radiofrequency catheter ablation for supraventricular tachycardia: should it be used in infants and small children? Circulation 1994;90:639-41.

26 Saul JP, Hulse E, Papagiannis J, et al. Late enlargement of radiofrequency lesions in infant lambs: implications for ablation procedures in small children. Circulation 1994;90:492-9. 